\title{
Different Dietary Protein Sources in Low-Protein Diets Induces Changes in Antioxidant Capacity, Immunity, Fecal Microbiota and Metabolites of Weaned Piglets
}

\section{Lianhua Zhang}

China Agricultural University

Xiangshu Piao ( $\sim$ piaoxsh@cau.edu.cn )

China Agricultural University https://orcid.org/0000-0001-8191-3028

\section{Research}

Keywords: Protein sources, Antioxidant capacity, Immunity, Bacterial community, Metabolites, Piglets

Posted Date: January 15th, 2021

DOI: https://doi.org/10.21203/rs.3.rs-143525/v1

License: (c) (1) This work is licensed under a Creative Commons Attribution 4.0 International License. Read Full License 


\section{Abstract}

Background: The inclusion of high-quality proteins and the protein restriction are commonly used in swine production. Our study was conducted to evaluate the effects of hydrolyzed wheat protein (HWP), fermented soybean meal (FSBM), and enzyme-treated soybean meal (ESBM) in low-protein diets on antioxidant capacity, immunity, fecal microbiota and metabolites of weaned piglets.

Methods: A total of 144 weaned piglets were randomly assigned to 3 dietary treatments with 6 replications of 8 piglets per pen. The experiment was divided into phase 1 (days 0-14) and phase 2 (days 15-28). The dietary treatments contained $15.90 \%$ HWP, 15.80\% FSBM, and 15.10\% ESBM in phase 1, and $7.90 \%$ HWP, $7.80 \%$ FSBM, and 7.50\% ESBM in phase 2, respectively.

Results: The ADG of piglets in the ESBM group was higher $(P<0.05)$ than HWP and FSBM during days 128. Compared with HWP and FSBM, piglets in ESBM had higher $(P<0.05)$ serum levels of FRAP and SOD on days 14 , as well as higher $(P<0.05)$ serum FRAP level on days 28. Piglets in ESBM had lower $(P<$ 0.05 ) serum levels of DAO and IL-1 $\beta$ than HWP on day 28 . ESBM significantly increased the abundance of Bacteroidetes, Oscillospiraceae and Christensenellaceae and decreased the Clostridiaceae abundance in the feces compared with HWP and FSBM. The PICRUSt analysis revealed that the number of gene tags involved in valine, leucine and isoleucine degradation and lysine degradation in ESBM were lower $(P<$ $0.05)$ than HWP and FSBM. Piglets in ESBM had higher $(P<0.05)$ fecal butyrate content compared with FSBM, and ESBM tended to decrease $(P=0.076)$ fecal cadaverine level.

Conclusions: Overall, ESBM had advantages over HWP and FSBM in improving antioxidant capacity, immunity, and fecal microbiota and metabolites of weaned piglets.

\section{Background}

In pig industry, weaning induces intestinal disturbances and results in continuous impairment of intestinal barrier function, intestinal inflammation, and oxidative damage [1, 2]. Weaned piglets are suddenly forced to undergo a transition from a highly digestible milk to less digestible, plant-based solid diets containing complex protein [3], which could result in maldigestion, diarrhea or even death. Therefore, it is of importance to supply high-quality proteins in diets to alleviate weaning stress in piglets. Animal protein sources including fish meal have been widely used in the diets of weaned piglets as a source of readily digestible, high quality protein. However, high price, limited supplies, and unstable quality of animal protein sources make the nutritionist explore alternative to animal protein sources for weaned piglets. Soybean meal (SBM) is a poorly tolerated protein ingredient for the gastrointestinal tract of weaned piglet as result of various anti-nutritional factors including glycinin, $\beta$-conglycinin and trypsin inhibitor, which leads to digestive disorders, immune responses and poor performance $[3,4]$. Fermentation or bioprocessing of SBM including fermented soybean meal (FSBM), enzyme-treated soybean meal (ESBM) has been shown to reduce the contents of antinutritional factors such as glycinin, $\beta$-conglycinin and trypsin inhibitor, as well as improve growth rate of weaned piglets [3]. Moreover, 
hydrolyzed wheat protein (HWP) is used as a practical, functional protein source to improve growth performance of weaned piglets due to its glutamine and a variety of biologically active-wheat peptides [5, 6].

The shortage of dietary protein resources and serious environmental contamination have been the main restrictive factors in the sustainable development of pig industry. Recent studies have reported that lowprotein diets supplemented with crystalline amino acids could save protein resources, decrease nitrogen emission and feed costs of pigs as well as reduce the incidence of intestinal injury without influencing pig performance in comparison with traditional diets [7-9]. There is an emerging interest on the relationship between protein ingredients and gut health. The gastrointestinal tract of pigs is colonized with a large and diverse group of microbial community that could confer multitudinous physiological functions to the host, such as metabolism of nutrients, production of short-chain fatty acids (SCFAs), and development of intestinal mucosa [10-12]. Dietary protein is mainly decomposed into peptides and free amino acids in the foregut under the catalytic reaction of proteases and peptidases, and the fermentation of undigested and endogenous protein takes place in the hindgut $[13,14]$. The degradation of undigested protein in the hindgut could produce a variety of beneficial metabolites including SCFAs or branchedchain fatty acids (BCFAs) [15], and some potentially toxic products such as ammonia, biogenic amines, phenolic and indolic compounds [16]. Therefore, low-protein diets and better digestibility of protein could decrease transfer of the undigested protein into the hindgut and reduce production of potentially toxic products from microbial metabolism.

Although plant protein sources including HWP, FSBM and ESBM have been widely used in the diets of weaned piglets, little work has been done on comparing the effects of these different protein sources in low-protein diets on immune function and protein fermentation in the hindgut of piglets. The objective of this study was to compare the effects of different dietary protein sources such as HWP, FSBM and ESBM in low-protein diets on immunity, fecal microbiota and metabolites of weaned piglets.

\section{Materials And Methods}

All animal procedures used in this experiment were reviewed and approved by the Institutional Animal Care and Use Committee of China Agricultural University (Beijing, China). The study was conducted at the China Agricultural University Animal Experimental Base (Hebei, China). The three protein sources used in this study were HWP (hydrolyzed wheat protein; JPC56, Joosten, Weert, The Netherlands), FSBM (fermented soybean meal; Yuanyao Biotechnology Co., LTD, Jiangsu, China) and ESBM (enzyme-treated soybean meal; HP300, Hamlet Protein, Horsens, Denmark).

\section{Animals, diets, and experimental design}

A total of 144 Duroc $\times$ Landrace $\times$ Large White weaned piglets, with an initial body weight (BW) of $7.75 \pm$ $0.96 \mathrm{~kg}$, were randomly assigned to 3 treatments with 6 replications of 8 piglets ( 4 barrows and 4 gilts) per pen. The whole experimental period lasted for $28 \mathrm{~d}$, and contained phase 1 (days 0-14) and phase 2 (days 15-28). The dietary treatments included (1) HWP diet: 15.90\% JPC56 replacing soybean meal in 
phase 1 and $7.90 \%$ JPC56 replacing soybean meal in phase 2; (2) FSBM diet: 15.80\% FSBM replacing soybean meal in phase 1 and 7.80\% FSBM replacing soybean meal in phase 2; (3) ESBM diet: 15.10\% HP300 replacing soybean meal in phase 1 and $7.50 \%$ HP300 replacing soybean meal in phase 2 . The experimental diets had similar level of crude protein (CP, 18.0\%). All diets were formulated to meet or exceed the nutrient requirements of National Research Council (NRC 2012) [17] for nursery pigs ranging from 7 to $11 \mathrm{~kg}$ (or 11 to $25 \mathrm{~kg}$ ) BW and fed as mash (Table 1).

All piglets were housed in an commercial flat-deck pens with duckbill drinkers, adjustable feeders and plastic slatted floors. Feeds and water were provided ad libitum for piglets. The room temperature and relative humidity were maintained at $24^{\circ} \mathrm{C}$ to $26^{\circ} \mathrm{C}$ and $60 \%$ to $70 \%$, respectively. Piglet were weighed individually after fasting on days 14 and 28 to calculate average daily gain (ADG), average daily feed intake (ADFI) and feed conversion ratio (FCR). Fecal score were determined by clinical signs of fecal consistency every day based on the methods described by Pan et al. [18] and a scoring system was applied to indicate the presence and severity of diarrhea as following: $1=$ hard feces; 2 = slightly soft feces; 3 = soft, partially formed feces; 4 = loose, semiliquid feces; and 5 = watery, mucous-like feces.

\section{Sample collection and preparation}

All diet samples were collected and stored at $4^{\circ} \mathrm{C}$ for further chemical analysis. On days 14 and 28, fasting blood samples (approximately $10 \mathrm{~mL}$ ) were collected from 1 piglet per pen (closest to the average body weight of each pen) via the jugular vein into vacutainer tubes (Greiner Bio-One GmbH, Kremsmunster, Austria). Serum samples were obtained by centrifugation at $3000 \times g$ for $10 \mathrm{~min}$ at $4^{\circ} \mathrm{C}$, and then stored at $-20^{\circ} \mathrm{C}$ until analysis of serum parameters. From d 26 to 28 , approximately $200 \mathrm{~g}$ of feces was collected from each pen for $3 \mathrm{~d}$ and stored at $-20^{\circ} \mathrm{C}$. Then the $3 \mathrm{~d}$ collection of feces was pooled by pen and dried at $65^{\circ} \mathrm{C}$ for $72 \mathrm{~h}$ to determine apparent total tract digestibility (ATTD) of nutrients. All samples were ground to pass through a 1-mm screen (40 mesh) before analysis. At the end of experiment, six fresh fecal samples (1 piglet per pen) in each treatment were rapidly frozen in liquid nitrogen, and then stored at $-80^{\circ} \mathrm{C}$ for further analysis of intestinal bacteria, SCFAs and nitrogen metabolites.

\section{Chemical analysis}

Diets and feces were analyzed in duplicate for the contents of dry matter (DM, method 934.01) and crude protein (CP, method 990.03) according to the Association of Official Analytical Chemists (AOAC, 2006) [19]. Gross energy (GE) was determined by an Automatic Energy Analyzer (Parr 1281, Moline, IL, USA). The chromium ( $\mathrm{Cr}$ ) content in the diets and feces was analyzed using an Atomic Absorption Spectrophotometer (Z-5000; Hitachi, Tokyo, Japan) according to the procedure of Williams et al. [20]. ATTD was determined by the equation as follows: digestibility $(\%)=100-\left[\left(\mathrm{Cr}_{\text {diet }} \times\right.\right.$ Nutrient $\left._{\text {feces }}\right) /\left(\mathrm{Cr}_{\text {feces }}\right.$ $\times$ Nutrient $\left.\left._{\text {diet }}\right)\right] \times 100$. The levels of advanced oxidation protein products (AOPP) and ferric reducing ability of plasma (FRAP) in serum were assessed using commercially available ELISA test kits (Zhongshang Boao Biotechnology Co., Ltd., Beijing, China). The contents of malondialdehyde (MDA), superoxide 
dismutase (SOD), catalase (CAT) and glutathione peroxidase (GSH-Px), diamine oxidase (DAO) and endotoxin in serum were measured using assay kits according to the manufacturer's protocols (Nanjing Jiancheng Bioengineering Institute, Jiangsu, China). Serum D-lactate level was measured by an ELISA kit (Luyuan Byrd Biotechnology Co., Ltd., Beijing, China). The levels of immunoglobulin G (IgG), immunoglobulin $\mathrm{A}(\lg \mathrm{A})$ and immunoglobulin $\mathrm{M}(\mathrm{IgM})$ in serum were measured using commercially available kits (Leadman Biochemistry Co., Ltd., Beijing, China). The concentrations of interleukin-1 $\beta$ (IL$1 \beta)$, interleukin-6 (IL-6) and tumour necrosis factor- $a$ (TNF- $\alpha$ ) in serum were determined using ELISA kits (Kangjia Hongyuan Biotechnology Co., Ltd., Beijing, China).

\section{DNA extraction, PCR amplification, Illumina MiSeq sequencing and data analysis}

Microbial DNA was extracted from the fecal samples using the DNA Kit (Omega Bio-tek, Norcross, GA, USA). The integrity of DNA was assessed by agarose gel electrophoresis. Isolated DNA was used as a template for amplification of the 16S rRNA gene V3-V4 region using universal primers 338F (5'-barcodeACTCCTRCGGGAGGCAGCAG-3') and 806R (5'-GGACTACCVGGGTATCTAAT-3'), where barcode is an eightbase sequence unique to each sample. After purification, amplicons were pooled and paired-end sequenced on the Illumina MiSeq platform (Illumina Inc., San Diego, CA, USA). Raw fastq files were demultiplexed, and quality-filtered using QIIME(version1.17). Operational taxonomic units (OTUs) were clustered with $97 \%$ similarity cutoff using UPARSE and chimeric sequences were removed using UCHIME. The Ribosomal Database Project (RDP) Classifier (http://rdp.cme.msu.edu/) was used to analyze the taxonomy of each $16 \mathrm{~S}$ rRNA gene sequence with confidence greater than $70 \%$. The Venn diagram was present based on the occurrence of shared and unique OTUs among different treatments. a-diversity including observed species, Chao, Ace, Shannon, Simpson, Good's coverage was performed using MOTHUR (v.1.31.2). The Unifrac metric was used to calculate $\beta$-diversity and UPGMA tree was displayed by QIIME (v1.80) based on Unweighted UniFrac distances of the OTUs community. Principal component analysis (PCA) was conducted by R software (v3.1.1) according to the OTUs among different treatments. LefSe analysis was performed to identify the biomarkers for microbial communities from phylum to genus based on the relative abundance of annotated taxonomic profiling.

\section{Predictive metabolic functions of microbial communities}

Predictive metabolic functions of the bacterial community in the fecal samples were conducted by Phylogenetic Investigation of Communities by Reconstruction of Unobserved States (PICRUSt) based on 16S rRNA gene sequencing [21]. OUTs were used to predict functional profiling of microbial communities by referencing the Kyoto Encyclopedia of Genes and Genome (KEGG) orthology database [22].

\section{Measurements of SCFAs and BCFAs by high performance ion chromatography}

The fecal samples were prepared for analyzing SCFAs and BCFAs as previously described by He et al. [23]. Samples $(0.5 \mathrm{~g})$ were weighed and dissolved in $8 \mathrm{~mL}$ of ultrapure water. After ultrasound for $30 \mathrm{~min}$, fecal samples were centrifuged at $3000 \times \mathrm{g}$ for $5 \mathrm{~min}$. The supernatant was diluted (1:50) and then filtered through a $0.22 \mu \mathrm{m}$ filter. Extracted sample solution $(25 \mu \mathrm{L})$ was analyzed for SCFAs (acetate, 
propionate, butyrate), BCFAs (isobutyrate, valerate and isovalerate) using a high performance ion chromatography system (DIONEX ICS-3000, Thermo Fisher, Waltham, MA, USA). The SCFAs and BCFAs were separated by an AS11 analytical column $(250 \times 4 \mathrm{~mm})$ and an AG11 guard column using potassium hydroxide. The concentration of SCFAs and BCFAs were expressed as $\mathrm{mg} / \mathrm{g}$ of feces.

\section{Analysis of ammonia nitrogen in feces}

The content of ammonia nitrogen $\left(\mathrm{NH}_{3}-\mathrm{N}\right)$ was analyzed based on the method as described by Chen et al. [24]. In short, $0.5 \mathrm{~g}$ of samples was dissolved in $5 \mathrm{~mL}$ of ammonia-free water and centrifuged at $5000 \times \mathrm{g}$ for $15 \mathrm{~min}$. Next, $19 \mathrm{~mL}$ of ammonia-free water and $1 \mathrm{~mL}$ of potassium sodium tartrate was added into 1 $\mathrm{mL}$ of collected supernatant in a 50-mL sterile tube, followed by the addition of $1.5 \mathrm{~mL}$ of Nessler's reagent. The absorbance of final mixture was determined at $420 \mathrm{~nm}$ against ammonia-free water using a UV-vis Spectrophotometer (MAPADA, Shanghai, China).

\section{Determination of biogenic amines by high performance liquid chromatography}

The concentrations of biogenic amines (putrescine, cadaverine and spermine) were determined by high performance liquid chromatography (HPLC) as previously described by Li et al. [25] with modification. Briefly, $0.5 \mathrm{~g}$ of fecal samples was added into a 2-mL centrifuge tube and mixed with $1 \mathrm{~mL}$ of trichloroacetic acid. The mixture was centrifuged at $3600 \times \mathrm{g}$ for $10 \mathrm{~min}$, the supernatants were then vortexed with the same volume of $n$-hexane for $5 \mathrm{~min}$. The aqueous phase was extracted again in the same manner. Internal standard $(20 \mathrm{~mL})$ was added into the extracts, followed by the addition of $1.5 \mathrm{~mL}$ of saturated sodium bicarbonate, $1 \mathrm{~mL}$ of dansyl chloride, and $1 \mathrm{~mL}$ of sodium hydroxide. The mixture was subsequently heated at $60^{\circ} \mathrm{C}$ for 45 min with occasional shaking. Next, $100 \mu \mathrm{L}$ of ammonia was added into the mixture and kept at $40^{\circ} \mathrm{C}$ in a water bath under $\mathrm{N}_{2}$ condition. Finally, the analyzed sample was produced by adding acetonitrile to the residue. Each sample $(20 \mu \mathrm{L})$ was injected into Agilent HPLC 1200 series equipped with a reversed-phase ZORBAX 80 A Extend-C18 column $(250 \mathrm{~mm} \times 4.6 \mathrm{~mm}$ i.d.; 5 $\mu \mathrm{m}$ particle size, Agilent, Santa Clara, USA). The flow rate, wavelength and column temperature were set at $1.0 \mathrm{~mL} / \mathrm{min}, 254 \mathrm{~nm}$ and $40^{\circ} \mathrm{C}$, respectively. The concentration of biogenic amines were expressed as $\mu \mathrm{g} / \mathrm{g}$ of feces.

\section{Statistical analysis}

Data analyses of growth performance, serum parameters, SCFA concentrations, and nitrogen metabolites were analyzed by One-Way ANOVA using the GLM procedure of SAS 9.4 (SAS Inst. Inc., Cary, NC), with results presented as mean \pm SEM. Differences in the fecal score were determined by the $\chi^{2}$ contingency test. Microbiota diversity metrics were conducted from standardized OTU reads using R software (version 3.2.2). The relative abundance of fecal microbiota composition was analyzed by the Kruskal-Wallis method. Differences were considered significant at $p<0.05$, and tendency was declared with $0.05 \leq p<$ 0.10 . 


\section{Growth performance and fecal score}

As shown in Table 2, piglets in the ESBM group had higher $(P<0.05)$ BW on days 14 and 28 than that of piglets in the HWP and FSBM groups. The ADG of piglets in the ESBM group was increased $(P<0.05)$ compared with piglets in the HWP and FSBM groups during days 1-14 and 1-28, and the ADG of piglets in the ESBM group was increased $(P<0.05)$ compared with the HWP group during days 15-28. The ADFI of piglets in the HWP group was lower $(P<0.05)$ compared with the FSBM and ESBM groups. Piglets in the ESBM group had lower $(P<0.05)$ FCR than the HWP and FSBM groups during days $1-14$, and had lower $(P<0.05)$ FCR than the FSBM group during days 1-28. No difference was observed for fecal score among all dietary treatments.

\section{Nutrient digestibility}

Piglets fed with ESBM or HWP had higher $(P<0.05)$ ATTD of CP compared with piglets fed with FSBM (Fig. 1). No difference was observed for ATTD of GE among dietary treatments.

\section{Serum oxidative status}

Piglets in the ESBM group had higher $(P<0.05)$ serum FRAP level than that of piglets in the HWP and FSBM groups on days 14 and 28 (Table 3 ). Serum activity of SOD was highest in piglets fed with ESBM diet on days 14 and 28. No significant differences were observed for serum levels of AOPP, MDA, CAT and GSH-Px among all dietary treatments.

\section{Serum DAO, endotoxin and D-lactate}

Piglets in the ESBM group had lower $(P<0.05)$ serum DAO level than that of piglets in the HWP group on day 28 (Table 4). No differences were observed for serum levels of endotoxin and D-lactate among treatments.

\section{Concentrations of inflammatory cytokines and immunoglobulins in serum}

As shown in Table 5, ESBM tended to decrease concentrations of IL-1 $(P=0.060)$ and IL-6 $(P=0.070)$ in serum of piglets on day 14. Piglets in the ESBM group had lower $(P<0.05)$ serum IL-1 $\beta$ level compared with the HWP group on day 28. No significant differences were observed for serum levels of IgA, IgG and IgM among all dietary treatments.

\section{Fecal bacterial community}

The OUT Venn analysis identified 47, 34 and 63 unique OTUs in the HWP, FSBM and ESBM groups, respectively (Fig. 2A). The Shannon index was higher $(P<0.05)$ in the fecal samples of the ESBM group compared with the HWP and FSBM groups, and the Simpson index was lower $(P<0.05)$ in the fecal samples of the ESBM group compared with the FSBM group (Fig. 2B). Principal component analysis (PCA) plot based on the OUT level showed that greater variations were observed in the fecal samples of the ESBM group compared with the HWP and FSBM groups, and the HWP and FSBM groups had similar 
microbial communities (Fig. 3A). The UPGMA tree showed that significant differences in the structure of fecal microbiota among different treatments, indicating that the protein sources had important impacts on fecal microbial communities (Fig. 3B).

At the phylum level, the dominant bacteria were Firmicutes and Bacteroidetes, which accounted for $90 \%$ (Fig. 4A). The abundance of Bacteroidetes in the fecal samples of the ESBM group was higher $(P<0.05)$ compared with the HWP and FSBM groups (Fig. 5A). At the family level, the predominant families within the Firmicutes phylum consisted of Christensenellaceae, Clostridiaceae, Erysipelotrichaceae, Lachnospiraceae, Lactobacillaceae, Oscillospiraceae, Peptostreptococcaceae, and Streptococcaceae, while Muribaculaceae was the dominant family in the Bacteroidetes phylum (Fig. 4B). The abundance of Clostridiaceae in the fecal samples of the ESBM group was lower $(P<0.05)$ than the HWP and FSBM groups, and ESBM increased $(P<0.05)$ the abundances of Oscillospiraceae and Christensenellaceae compared with HWP and FSBM (Fig. 5B).

LEfSe analysis was performed to identify specific bacteria that were characteristic for piglets among three dietary treatments (Fig. 6). The results showed that 30 different OTUs among treatments (Fig. 6A). Among the different OTUs, 8 of these OTUs were higher in the HWP group, 4 of these OTUs were higher in the FSBM group, and 18 of these OTUs were higher in the ESBM group. A great abundance of Roseburia, Holdemanella, unclassified_f_Erysipelotrichaceae, unclassified_p_Firmicutes, and norank_f_Prevotrllaceae in the HWP group, Eubacterium_ventriosum_group, Clostridiales, Clostridiaceae, and Clostridium_sensu_stricto_1 in the FSBM group, Lachnospiraceae_NK4A136_group, Oscillospiraceae, UCG_005, UCG_002, Oscillibacter, NK4A214_group, norank_o_Oscillospirales, Christensenellales, Christensenellaceae, Christensenellaceae_R_7_group, Clostridia_vaclinBB60_group, norank_o_Clostridia_vadinBB60_group, norank_f_norank_o_Clostridia_vadinBB60_group, Bacteroidetes, Bacteroidia, Bacteroidales, and Corynebacteriales in the ESBM group were observed (Fig. 6B).

\section{Prediction on amino acid metabolism of bacterial communities using PICRUSt}

Ten pathways associated with amino acid metabolism were shown in Fig. 7, including tryptophan metabolism, cysteine and methionine metabolism, phenylalanine metabolism, glycine, serine and threonine metabolism, tyrosine metabolism, valine, leucine and isoleucine degradation, lysine degradation, arginine and proline metabolism, histidine metabolism, as well as alanine, aspartate and glutamate metabolism. The number of the genes associated with valine, leucine and isoleucine degradation and lysine degradation in the ESBM group decreased $(P<0.05)$ compared with the HWP and FSBM groups. No differences were observed for other pathways associated with amino acid metabolism among all dietary treatments.

\section{Fermentation metabolites of fecal samples}

The concentration of SCFAs in the fecal samples of piglets fed diets with different dietary protein sources was shown in Table 6. Butyrate concentration in the fecal samples of the ESBM group was higher $(P<$ 0.05) than that of the FSBM group. No difference was observed for $\mathrm{NH}_{3}-\mathrm{N}$ among all dietary treatments 
(Table 7). The content of cadaverine in the fecal samples of the ESBM group tended to be lower $(P=$ 0.076) compared with other groups.

\section{Discussion}

Restriction on the use of animal products in livestock has aroused interest in replacing animal protein sources with plant protein sources, and this need becomes more pronounced when the diet is focused on weaned piglets. In pig industry, plant protein sources such as HWP, FSBM and ESBM have been widely used in the diets of weaned piglets to improve growth performance $[3,5,6,26]$. However, little information was available towards comparing the effect of HWP, FSBM and ESBM on growth performance of weaned piglets. In this study, we found that piglets fed with ESBM had higher ADG than that of piglets fed with HWP and FSBM, indicating that piglets in ESBM had a higher growth rate compared with other diets. However, there were different reasons for lower ADG in piglets fed with HWP or FSBM compared with ESBM. Our result showed that no difference was observed for FCR between HWP and ESBM groups, but piglets fed with HWP had lower ADFI than that of piglets fed with ESBM during the whole period, indicated that lower ADFI in HWP may contribute to lower ADG in HWP compared with ESBM group. The inclusion of wheat gluten could decrease ADFI by increasing digesta viscoelasticity and affecting the transit of digesta [27]. In addition, no difference was observed for ADFI between FSBM and ESBM groups, but piglets fed with FSBM had higher FCR than that of piglets fed with ESBM, indicated that higher FCR in FSBM may contribute to lower ADG in FSBM compared with ESBM group. However, Ma et al. [3] showed that there was no significant difference for FCR between FSBM and ESBM groups. The inconsistency may be explained by the different experimental condition, diet composition, and inclusion level. Feed is the main source of energy and protein for animal maintenance and growth requirement. The digested and absorbed nutrients by gastrointestinal tract provide energy and amino acid for animals maintenance and growth via biological oxidation and metabolism [28]. In this study, piglets fed with FSBM had higher ADFI than that of piglets fed with HWP. However, no difference was observed for ADG between HWP and FSBM groups. Interestingly, piglets fed with HWP had higher ATTD of CP compared with piglets fed with FSBM, which may explain no difference for ADG between HWP and FSBM groups.

Weaning could induce oxidative stress [3], which is considered to be an imbalance process between the production rate of reactive oxygen species (ROS) and their clearance via the antioxidant system [29]. Antioxidant capacities in serum reflect the host's capacity to respond to endogenous oxidative damage, and improving the antioxidant capacities has positive effects on oxidative stress [30]. Normally, excessive oxidative radicals are eliminated by antioxidant systems including nonenzymatic antioxidant defensive components and a series of antioxidant enzymes. FRAP is considered a useful indicator of "the total antioxidant power" of plasma, reflecting the combined antioxidant effect of non-enzymatic compounds of endogenous and exogenous origin in biological fluids [31]. In this study, piglets fed with ESBM had higher serum FRAP level than that of piglets fed with HWP or FSBM, indicating that ESBM had an advantage over HWP and FSBM in improving non-enzymatic antioxidant defense. Weaning stress could decrease serum concentrations of SOD and GSH-Px, as well as improve serum MDA and hydrogen 
peroxide $\left(\mathrm{H}_{2} \mathrm{O}_{2}\right)$ contents in piglets [32]. Our results showed that piglets fed with ESBM had higher serum SOD concentration than that of piglets fed with HWP or FSBM. It has been known that SOD acts as the first line of defense against excessive oxidative radicals via catalyzing the dismutation of the endogenous superoxide radical to $\mathrm{H}_{2} \mathrm{O}_{2}$, which is decomposed into $\mathrm{H}_{2} \mathrm{O}$ and $\mathrm{O}_{2}$ by GSH-Px and CAT [33]. Our result suggested that ESBM could have higher ability to improve antioxidant capacity via activating the enzymatic and nonenzymatic antioxidant defensive systems compared with HWP or FSBM.

The intestinal epithelial barrier consists mainly of a single layer of enterocytes and intercellular multiprotein complexes, which can maintain intestinal homeostasis by preventing the flows of pathogens, toxins, and antigens from the gut lumen into the circulating system [8,34]. Weaning stress could induce imperfect and impaired gastrointestinal tract, which is associated with increased permeability, resulting in translocation of pathogens through the intestinal epithelial cells into systemic circulation [8]. DAO, endotoxin and D-lactate are intestinal permeability-related biomarkers and their levels in serum could reflect the intestinal barrier function [35]. In this study, HWP increased serum DAO concentration on day 28 compared with ESBM, indicating that piglets in the ESBM group had better intestinal barrier function. Weaning process leads to intestinal inflammation and promotes the release of pro-inflammatory cytokines, which can explain the stunted growth performance and intestinal disorders of piglets [36]. Numerous pro-inflammatory cytokines including IL-1 $\beta$, IL-6, and TNF- $\alpha$ are involved in the regulation of the inflammatory response and their levels could reflect the inflammatory status in the body [35]. Our result showed that ESBM decreased serum levels of IL-1 $\beta$ and IL- 6 compared with HWP and FSBM, indicating that ESBM was superior to HWP and FSBM in alleviating weaning stress-induced inflammatory response.

The intestinal microbiota of swine is the diverse and dense community of microorganisms that could confer multifarious physiological functions to the host such as nutrient metabolism and development of the intestinal mucosa [8]. Diet composition, particularly macronutrients such as carbohydrates and protein, reflects the substrates available for the intestinal microbiota and influences microbial composition and metabolism, which in turn could improve animal performance and health [25]. Normally, the diet ingredients are digested and absorbed in the foregut, and the undigested residues as well as endogenous compounds flow into the hindgut for microbial fermentation, which modulates the intestinal microbiota and bacterial metabolites $[8,37]$. Carbohydrates fermentation is beneficial to intestinal epithelial cells due to the production of SCFAs [25,38], while protein fermentation has adverse impacts on intestinal health due to potentially toxic metabolites such as ammonia, biogenic amines, and aromatic compounds that are related to proliferation of pathogenic bacteria $[8,25]$. In this study, we focus on the fecal microbiota composition and metabolites in piglets fed with different dietary protein sources including HWP, FSBM, and ESBM. The a-diversity is regarded as indicators of functional resilience of microbial ecosystems in the intestine, including species richness (Observed species, Chao, and Ace) and species diversity (Shannon, Simpson, and Good's coverage) [39]. Our results showed that no changes were observed for species richness of the fecal samples in piglets fed with HWP, FSBM, and ESBM. However, ESBM increased Shannon index in the fecal samples of piglets compared with HWP and FSBM, 
and ESBM decreased Simpson index in the fecal samples of piglets compared with FSBM, revealing that ESBM had an advantage over HWP and FSBM in improving fecal bacterial diversity without affecting bacterial richness. The $\beta$-diversity by PCA and UPGMA tree analysis showed that fecal microbiota responded differently to dietary protein sources. The reason for greater variations in the fecal microbiota structure of ESBM compared with other groups was unclear, but fecal microbiota structure of HWP and FSBM was similar, which may partly explain similar growth performance of piglets. At the phylum level, Firmicutes and Bacteroidetes were the most predominant bacterial phyla in the fecal samples, which is similar to the results conducted by Zhao et al. [40]. In this study, the addition of ESBM to weaned piglets significantly increased the relative abundance of Bacteroidetes in the fecal samples of piglets compared with HWP and FSBM. The phylum Bacteroidetes are considered as effective degraders for plant polysaccharides and other recalcitrant organic carbon and nitrogen sources [41]. The higher abundance of Bacteroidetes are known to help shape the metabolic milieu of the intestinal ecosystem and prevent diarrhea because they could interact with the intestinal immune system, suggesting the relationship between early bacterial colonization and intestinal immune maturation after weaning [42]. Although no difference was observed for fecal score among different protein sources, higher abundance of Bacteroidetes in piglets fed with ESBM could contribute to improvement in the intestinal immunity and further promote growth performance of piglets. Down to the family level, piglets fed with ESBM had lower abundance of Clostridiaceae and higher abundance of Oscillospiraceae and Christensenellaceae in the fecal samples of piglets compared with HWP and FSBM. In our study, these findings were also confirmed by LEfSe analysis, which can identify unique high-dimensional biomarkers for analyzed microbial communities [43]. Certain bacterial species, from the family Clostridiaceae, were implicated in poor performance [25]. In addition, Clostridiaceae comprises certain pathobionts. For instance, Clostridium perfringens, belonging to the family Clostridiaceae, may not necessarily compromise the health of the pig, but they could have the potential to zoonotic diseases if excreted in the form of feces [44]. In previous studies, the Oscillospiraceae and Christensenellaceae families have been shown to include microorganisms with potential health benefits and contribute to the formation of secondary bile acids that are known to protect against infection with Clostridium difficile [45, 46]. Butyrate is one of the important microbial metabolites that could provide energy for colonocytes and has beneficial influences on epithelium proliferation and differentiation [47]. The family Christensenellaceae is also considered as a butyrate producer and play a key role in maintaining gastrointestinal tract structure and function [34], which may be an explanation for the increased butyrate content in the fecal samples of piglets fed with ESBM. Together, these findings suggested that dietary protein sources could differentially influence fecal bacterial community of weaned piglets.

Dietary carbohydrates and proteins can be fermented in the colon to produce SCFAs and BCFAs [14]. SCFAs are derived from fermentation of dietary carbohydrates and protein, and deamination of valine, leucine, and isoleucine could produce BCFAs [48]. In this study, no difference was observed for BCFAs among all dietary treatments. However, using the PICRUSt program to predict amino acid metabolism of bacterial communities, the number of gene tags involved in valine, leucine, and isoleucine degradation in the ESBM group were significantly decreased compared with HWP and FSBM groups. In this study, the 
reason for this result may be that the gene tags are primarily related to decarboxylation, and it is necessary to further investigate the relationship between dietary protein sources and the degradation of valine, leucine, and isoleucine. The microbial fermentation of amino acids in the hindgut generates some potentially toxic products including ammonia, biogenic amines, phenols, and indoles [16], which are closely related to the proliferation of pathogenic bacteria and depress the growth performance of pigs [14]. The low-protein diets supplemented with an adequate level of crystalline amino acids may deter the formation of protein fermentation products in the large intestine without diminishing the growth performance of pigs [7]. $\mathrm{NH}_{3}-\mathrm{N}$ is a toxic metablite produced by the deamination of amino acids, which can interfere with epithelial cell turnover and reduce growth performance of pigs [24, 25]. Our results showed that no difference was observed for $\mathrm{NH}_{3}-\mathrm{N}$ content in the fecal samples among all dietary treatments, indicating that microbial deamination of dietary and endogenous protein was not altered by the addition of HWP, FSBM, and ESBM to low-protein diets. However, ESBM tended to decrease fecal cadaverine content compared with HWP and FSBM, which revealed a decline in protein fermentation. Cadaverine is produced by microorganisms through the decarboxylation of lysine in the large intestinal lumen $[49,50]$. The PICRUSt analysis revealed that the number of gene tags involved in lysine degradation in the ESBM group were significantly decreased compared with HWP and FSBM groups. The result may contribute to explain decreased cadaverine content in the fecal samples of piglets fed with ESBM.

\section{Conclusions}

Different protein sources in low-protein diets could differentially modulate antioxidant capacity, immune function, and fecal bacterial community and metabolites of weaned piglets. ESBM had advantages over HWP and FSBM in improving growth performance and health of weaned piglets, which is mainly reflected by the increased $A D G$, the improved antioxidant status and immunity via increasing serum FRAP and SOD levels and decreasing serum DAO and IL- $1 \beta$ concentrations, and the improved fecal microbiota composition and metabolites including lower abundance of Clostridiaceae, higher abundance of Bacteroidetes, Oscillospiraceae and Christensenellaceae, enhanced fecal butyrate content and reduced protein fermentation.

\section{Abbreviations}

$A D F I$, average daily feed intake; ADG, average daily gain; AOPP, advanced oxidation protein products; BSFAs, branched-chain fatty acids; $B W$, body weight; CAT, catalase; $C P$, crude protein; DAO, diamine oxidase; DM, dry matter; ESBM, enzyme-treated soybean meal; FCR, feed conversion ratio; FRAP, ferric reducing ability of plasma; FSBM, fermented soybean meal; GE, gross energy; GSH-Px, glutathione peroxidase; HWP, hydrolyzed wheat protein; IgA, immunoglobulin $A$; IgG, immunoglobulin $G$; IgM, immunoglobulin M; IL-1 $\beta$, interleukin-1 $\beta$; IL-6, interleukin-6; LDA, linear discriminant analysis; MDA, malondialdehyde; $\mathrm{NH}_{3}-\mathrm{N}$, ammonia nitrogen; OUT, operational taxonomic units; SCFAs, short chain fatty acids; SOD, total superoxide dismutase; TNF-a, tumour necrosis factor-a. 


\section{Declarations}

Acknowledgements

Not applicable.

\section{Author's contributions}

LHZ and XSP designed the experiment. LHZ performed the experiment and statistical data analysis, and wrote the manuscript. XSP edited the manuscript. All authors have read and approved the final manuscript.

\section{Funding}

This research was supported by the National Natural Science Foundation of China (31772612), and the Beijing Municipal Natural Science Foundation (6202019).

\section{Availability of data and material}

All data generated or analyzed during this study are included in this published article.

\section{Ethics approval and consent to participate}

This experiment was approved by Committee of China Agricultural University Laboratory Animal Care and Use (Beijing, China).

\section{Consent for publication}

Not applicable.

\section{Competing interests}

The authors declare that they have no conflict of interest.

\section{References}

1. Hu R, He Z, Liu M, Tan J, Zhang H, Hou DX, et al. Dietary protocatechuic acid ameliorates inflammation and up-regulates intestinal tight junction proteins by modulating gut microbiota in LPS-challenged piglets. J Anim Sci Biotechnol. 2020;11:92.

2. Yin J, Wu MM, Xiao H, Ren WK, Duan JL, Yang G, et al. Development of an antioxidant system after early weaning in piglets. J Anim Sci. 2014;92:612-619.

3. Ma XK, Shang QH, Hu JX, Liu HS, Brøkner C, Piao XS. Effects of replacing soybean meal, soy protein concentrate, fermented soybean meal or fish meal with enzyme-treated soybean meal on growth 
performance, nutrient digestibility, antioxidant capacity, immunity and intestinal morphology in weaned pigs. Livest Sci. 2019;225:39-46.

4. Zheng L, Li D, Li ZL, Kang LN, Jiang YY, Liu XY, et al. Effects of Bacillus fermentation on the protein microstructure and anti-nutritional factors of soybean meal. Lett Appl Microbiol. 2017;65:520-526.

5. Wang XQ, You F, Gang S, Jiang QY, Yang JP, Zhang ZF. Effect of dietary supplementation with hydrolyzed wheat gluten on growth performance, cell immunity and serum biochemical indices of weaned piglets (Sus scrofa). Agr Sci China. 2011;10:938-945.

6. Han F, Wang Y, Wang W, Cheng F, Lu Z, Li A, et al. Effects of enzymatically hydrolyzed wheat gluten on growth performance, antioxidant status, and immune function in weaned pigs. Can J Anim Sci. 2017;97:574-580.

7. Wang Y, Zhou J, Wang G, Cai S, Zeng X, Qiao S. Advances in low-protein diets for swine. J Anim Sci Biotechnol. 2018;9:60.

8. Li R, Hou G, Jiang X, Song Z, Fan Z, Hou DX, et al. Different dietary protein sources in low protein diets regulate colonic microbiota and barrier function in a piglet model. Food Funct. 2019;10:6417-

9. Zhou L, Fang L, Sun Y, Su Y, Zhu W. Effects of the dietary protein level on the microbial composition and metabolomic profile in the hindgut of the pig. Anaerobe. 2016;38:61-

10. Zhang LH, Li M, Shang QH, Hu JX, Long SF, Piao XS. Effects of maternal 25-hydroxycholecalciferol on nutrient digestibility, milk composition and fatty-acid profile of lactating sows and gut bacterial metabolites in the hindgut of suckling piglets. Arch Anim Nutr. 2019;73:271-

11. Holman DB, Brunelle BW, Trachsel J, Allen HK. Meta-analysis to define a core microbiota in the swine gut. mSystems. 2017;2:e00004-

12. Kamada N, Kim YG, Sham HP, Vallance BA, Puente JL, Martens EC, et al. Regulated virulence controls the ability of a pathogen to compete with the gut microbiota. Science. 2012;336:1325-1329.

13. Fan P, Li L, Rezaei A, Eslamfam S, Che D, Ma X. Metabolites of dietary protein and peptides by intestinal microbes and their impacts on gut. Curr Protein Pept Sci. 2015;16:646-

14. Rist VT, Weiss E, Eklund M, Mosenthin R. Impact of dietary protein on microbiota composition and activity in the gastrointestinal tract of piglets in relation to gut health: a review. Animal. 2013;7:1067-1078.

15. Neis EP, Dejong $\mathrm{CH}$, Rensen SS. The role of microbial amino acid metabolism in host metabolism. Nutrients. 2015;7:2930-2946.

16. Portune KJ, Beaumont M, Davila A, Tomé D, Blachier F, Sanz Y. Gut microbiota role in dietary protein metabolism and health-related outcomes: the two sides of the coin. Trends Food Sci Technol. 2016;57:213-232.

17. Nutrient requirements of swine, 11th rev. ed. National Academy Press, Washington, DC. 2012.

18. Pan L, Ma XK, Wang HL, Xu X, Zeng ZK, Tian QY, et al. Enzymatic feather meal as an alternative animal protein source in diets for nursery pigs. Anim Feed Sci Technol. 2016;212:112-121.

19. Official Methods of Analysis, 18th ed. AOAC Int., Arlington, VA. 2006. 
20. Williams $\mathrm{CH}$, David DJ, lismaa $\mathrm{O}$. The determination of chromic oxide in feces samples by atomic absorption spectrophotometry. J Agric Sci. 1962;59:381-385.

21. Langille MG, Zaneveld J, Caporaso JG, McDonald D, Knights D, Reyes JA, et al. Predictive functional profiling of microbial communities using 16S rRNA marker gene sequences. Nat Biotechnol. 2013;31:814-821.

22. Kanehisa M, Goto S, Sato Y, Kawashima M, Furumichi M, Tanabe M. Data, information, knowledge and principle: Back to metabolism in KEGG. Nucleic Acids Res. 2014;42:D199-D205.

23. He B, Bai Y, Jiang L, Wang W, Li T, Liu P, et al. Effects of oat bran on nutrient digestibility, intestinal microbiota, and inflammatory responses in the hindgut of growing pigs. Int J Mol Sci. 2018;19:2407.

24. Chen J, Kang B, Jiang Q, Han M, Zhao Y, Long L, et al. Alpha-ketoglutarate in low-protein diets for growing pigs: effects on cecal microbial communities and parameters of microbial metabolism. Front Microbiol. 2018;9:1057.

25. Li R, Chang L, Hou G, Song Z, Fan Z, He X, et al. Colonic microbiota and metabolites response to different dietary protein sources in a piglet model. Front Nutr. 2019;6:151.

26. Ma XK, Shang QH, Wang QQ, Hu JX, Piao XS. Comparative effects of enzymolytic soybean meal and antibiotics in diets on growth performance, antioxidant capacity, immunity, and intestinal barrier function in weaned pigs. Anim Feed Sci Technol. 2019;248:47-58.

27. Blasco M, Fondevila M, Guada JA. Inclusion of wheat gluten as a protein source in diets for weaned pigs. Anim Res. 2005;54:297-306.

28. Chwalibog A, Tauson AH, Thorbek G. Energy metabolism and substrate oxidation in pigs during feeding, starvation and re-feeding. J Anim Physiol Anim Nutr. 2004;88:101-112.

29. Bhat AH, Dar KB, Anees S, Zargar MA, Masood A, Sofi MA, et al Oxidative stress, mitochondrial dysfunction and neurodegenerative diseases; a mechanistic insight. Biomed Pharmacother. 2015;74:101-110.

30. Wang Y, Xie Q, Sun S, Huang B, Zhang Y, Xu Y, et al. Probiotics-fermented Massa Medicata Fermentata ameliorates weaning stress in piglets related to improving intestinal homeostasis. Appl Microbiol Biotechnol. 2018;102:10713-10727.

31. Benzie IF, Strain JJ. The ferric reducing ability of plasma (FRAP) as a measure of "antioxidant power": the FRAP assay. Anal Biochem. 1996;239:70-76.

32. Zhu LH, Zhao KL, Chen XL, Xu JX. Impact of weaning and an antioxidant blend on intestinal barrier function and antioxidant status in pigs. J Anim Sci. 2012;90:2581-2589.

33. Bai K, Feng C, Jiang L, Zhang L, Zhang J, Zhang L, et al. Dietary effects of Bacillus subtilis fmbj on growth performance, small intestinal morphology, and its antioxidant capacity of broilers. Poult Sci. 2018;97:2312-

34. Shang Q, Liu H, Liu S, He T, Piao X. Effects of dietary fiber sources during late gestation and lactation on sow performance, milk quality, and intestinal health in piglets. J Anim Sci. 2019;97:4922-4933. 
35. Xiong W, Ma H, Zhang Z, Jin M, Wang J, Xu Y, et al. Icariin enhances intestinal barrier function by inhibiting NF-KB signaling pathways and modulating gut microbiota in a piglet model. Rsc Adv 2019;9:37947-37956.

36. Bomba L, Minuti A, Moisá SJ, Trevisi E, Eufemi E, Lizier M, et al. Gut response induced by weaning in piglet features marked changes in immune and inflammatory response. Funct. Integr. Genomics. 2014;14:657-671.

37. Zhu Y, Shi X, Lin X, Ye K, Xu X, Li C, et al. Beef, chicken, and soy proteins in diets induce different gut microbiota and metabolites in rats. Front Microbiol. 2017;8:1395.

38. Wong JM, de Souza R, Kendall CW, Emam A, Jenkins DJ. Colonic health: fermentation and short chain fatty acids. J Cli Gastroenterol 2006;40:235-243.

39. Zhang LH, Liu SJ, Li M, Piao XS. Effects of maternal 25-hydroxycholecalciferol during the last week of gestation and lactation on serum parameters, intestinal morphology and microbiota in suckling piglets. Arch Anim Nutr. 2020;74:445-461.

40. Zhao J, Liu P, Wu Y, Guo P, Liu L, Ma N, et al. Dietary fiber increases butyrate-producing bacteria and improves the growth performance of weaned piglets. J Agric Food Chem. 2018;66:7995-8004.

41. Yu T, Wang Y, Chen S, Hu M, Wang Z, Wu G, et al. Low-molecular-weight chitosan supplementation increases the population of Prevotella in the cecal contents of weanling pigs. Front Microbiol. 2017;8:2182.

42. Jakobsson HE, Abrahamsson TR, Jenmalm MC, Harris K, Quince C, Jernberg C, et al. Decreased gut microbiota diversity, delayed Bacteroidetes colonisation and reduced Th1 responses in infants delivered by caesarean section. Gut. 2014;63:559-566.

43. Segata N, Izard J, Waldron L, Gevers D, Miropolsky L, Garrett WS, et al. Metagenomic biomarker discovery and explanation. Genome Biol. 2011;12:R60.

44. Baer A, Miller M, Dilger A. Pathogens of interest to the pork industry: A review of research on interventions to assure food safety. Compr Rev Food Sci Food Saf. 2013;12:183-217.

45. Konikoff T, Gophna U. Oscillospira: a central, enigmatic component of the human gut microbiota. Trends Microbiol. 2016;24:523-524.

46. Chernevskaya E, Beloborodova N, Klimenko N, Pautova A, Shilkin D, Gusarov V, et al. Serum and fecal profiles of aromatic microbial metabolites reflect gut microbiota disruption in critically ill patients: a prospective observational pilot study. Crit Care. 2020;24:312.

47. Morrison DJ, Preston T. Formation of short chain fatty acids by the gut microbiota and their impact on human metabolism. Gut Microbes. 2016;7:189-200.

48. Fan P, Liu P, Song P, Chen X, Ma X. Moderate dietary protein restriction alters the composition of gut microbiota and improves ileal barrier function in adult pig model. Sci Rep. 2017;7:43412.

49. Davila AM, Blachier F, Gotteland M, Andriamihaja M, Benetti PH, Sanz Y, et al. Re-print of "Intestinal luminal nitrogen metabolism: role of the gut microbiota and consequences for the host". Pharmacol Res. 2013;69:114-126. 
50. Luo Z, Li C, Cheng Y, Hang S, Zhu W. Effects of low dietary protein on the metabolites and microbial communities in the caecal digesta of piglets. Arch Anim Nutr. 2015;69:212-226.

\section{Tables}

Table 1 Ingredient composition and nutrient levels of the experimental $\operatorname{diet}^{1}$ (\%, as-fed basis). 


\begin{tabular}{|c|c|c|c|c|c|c|}
\hline \multirow[t]{2}{*}{ Item } & \multicolumn{3}{|c|}{ Phase 1 (days 0-14) } & \multicolumn{3}{|c|}{ Phase 2 (days 15-28) } \\
\hline & HWP & FSBM & ESBM & HWP & FSBM & ESBM \\
\hline \multicolumn{7}{|l|}{ Ingredients } \\
\hline Corn & 39.53 & 39.22 & 40.16 & 37.21 & 37.12 & 37.54 \\
\hline Wheat & 20.00 & 20.00 & 20.00 & 20.00 & 20.00 & 20.00 \\
\hline Soybean meal & 5.00 & 5.00 & 5.00 & 16.00 & 16.00 & 16.00 \\
\hline HWP & 15.90 & & & 7.90 & & \\
\hline FSBM & & 15.80 & & & 7.80 & \\
\hline ESBM & & & 15.10 & & & 7.50 \\
\hline Whey power & 12.00 & 12.00 & 12.00 & 12.00 & 12.00 & 12.00 \\
\hline Soybean oil & 3.60 & 3.80 & 3.40 & 3.60 & 3.70 & 3.50 \\
\hline Dicalcium phosphate & 1.50 & 1.30 & 1.35 & 1.10 & 1.00 & 1.00 \\
\hline Limestone & 0.85 & 0.95 & 0.90 & 0.75 & 0.80 & 0.79 \\
\hline Salt & 0.30 & 0.30 & 0.30 & 0.30 & 0.30 & 0.30 \\
\hline L-Lysine $\mathrm{HCl}$ & 0.50 & 0.73 & 0.70 & 0.40 & 0.52 & 0.50 \\
\hline DL-Methionine & 0.02 & 0.15 & 0.25 & 0.06 & 0.11 & 0.17 \\
\hline L-Threonine & 0.25 & 0.23 & 0.21 & 0.16 & 0.15 & 0.14 \\
\hline L-Tryptophan & 0.05 & 0.02 & 0.13 & 0.02 & 0.00 & 0.06 \\
\hline Premix ${ }^{2}$ & 0.50 & 0.50 & 0.50 & 0.50 & 0.50 & 0.50 \\
\hline \multicolumn{7}{|l|}{ Nutrient levels } \\
\hline Digestible energy (kcal/kg) & 3399 & 3399 & 3399 & 3349 & 3350 & 3350 \\
\hline Dry matter ${ }^{3}$ & 90.06 & 89.97 & 90.02 & 89.93 & 89.86 & 89.91 \\
\hline Crude protein ${ }^{3}$ & 17.99 & 18.02 & 18.01 & 18.01 & 18.01 & 18.04 \\
\hline Crude fibre & 2.10 & 2.29 & 2.21 & 2.44 & 2.54 & 2.49 \\
\hline Total starch & 36.09 & 35.92 & 36.53 & 34.97 & 34.93 & 35.20 \\
\hline
\end{tabular}

${ }^{1} \mathrm{HWP}$, hydrolyzed wheat protein; FSBM, fermented soybean meal; ESBM, enzyme-treated soybean meal.

${ }^{2}$ Vitamin and mineral premix provided the following per kilogram of diet: $12,000 \mathrm{IU}$ vitamin A as vitamin A acetate, 2,500IU vitamin Das vitamin $D_{3}$, 30 IU vitamin $E$ as DL-a-tocopheryl acetate, $12 \mu \mathrm{g}$ of vitamin $B_{12}$, 
$3 \mathrm{mg}$ vitamin $\mathrm{K}$ as menadione sodium bisulfate, $15 \mathrm{mg}$ D-pantothenic acid as calcium pantothenate, 40 $\mathrm{mg}$ of nicotinic acid, $400 \mathrm{mg}$ choline as choline chloride, $30 \mathrm{mg} \mathrm{Mn}$ as manganese oxide, $80 \mathrm{mg} \mathrm{Zn}$ as zinc oxide, $90 \mathrm{mg}$ Fe as iron sulfate, $10 \mathrm{mg} \mathrm{Cu}$ as copper sulfate, $0.35 \mathrm{mg} \mathrm{I}$ as ethylenediamine dihydroiodide, and $0.3 \mathrm{mg}$ Se as sodium selenite.

${ }^{3}$ Analyzed value.

Table 2 The growth performance of piglets fed diets with different dietary protein sources ${ }^{1}$.

\begin{tabular}{|c|c|c|c|c|}
\hline Item & HWP & FSBM & ESBM & $P$-Value \\
\hline Initial BW (kg) & $7.75 \pm 0.42$ & $7.76 \pm 0.42$ & $7.76 \pm 0.42$ & 0.157 \\
\hline Day 14 BW(kg) & $11.18 \pm 0.54^{b}$ & $11.40 \pm 0.54^{b}$ & $11.75 \pm 0.60^{\mathrm{a}}$ & 0.002 \\
\hline Day 28 BW(kg) & $16.77 \pm 0.67^{b}$ & $17.20 \pm 0.86^{b}$ & $18.19 \pm 0.84^{a}$ & 0.001 \\
\hline \multicolumn{5}{|l|}{ Days 1-14 } \\
\hline$A D G(g / d)$ & $244.75 \pm 9.66^{b}$ & $259.77 \pm 10.31^{b}$ & $285.97 \pm 13.64^{a}$ & 0.002 \\
\hline $\operatorname{ADFI}(g / d)$ & $433.96 \pm 19.74^{b}$ & $456.88 \pm 23.35^{a}$ & $461.67 \pm 25.88^{a}$ & 0.025 \\
\hline FCR & $1.78 \pm 0.05^{a}$ & $1.76 \pm 0.04^{\mathrm{a}}$ & $1.61 \pm 0.03^{b}$ & 0.030 \\
\hline Fecal score & $2.32 \pm 0.10$ & $2.37 \pm 0.06$ & $2.29 \pm 0.07$ & 0.732 \\
\hline \multicolumn{5}{|l|}{ Days $15-28$} \\
\hline$A D G(g / d)$ & $399.66 \pm 16.72^{b}$ & $416.25 \pm 24.92^{a b}$ & $459.66 \pm 20.28^{a}$ & 0.047 \\
\hline $\operatorname{ADFI}(g / d)$ & $666.64 \pm 41.37^{b}$ & $731.96 \pm 47.79^{a}$ & $756.73 \pm 37.07^{a}$ & 0.023 \\
\hline FCR & $1.67 \pm 0.07$ & $1.76 \pm 0.03$ & $1.65 \pm 0.03$ & 0.170 \\
\hline Fecal score & $2.24 \pm 0.03$ & $2.20 \pm 0.05$ & $2.24 \pm 0.15$ & 0.958 \\
\hline \multicolumn{5}{|l|}{ Days 1-28 } \\
\hline $\operatorname{ADG}(\mathrm{g} / \mathrm{d})$ & $322.20 \pm 11.07^{b}$ & $337.54 \pm 15.93^{b}$ & $372.81 \pm 16.01^{a}$ & 0.001 \\
\hline $\operatorname{ADFI}(g / d)$ & $550.30 \pm 30.02^{b}$ & $594.42 \pm 34.57^{\mathrm{a}}$ & $609.20 \pm 30.78^{a}$ & 0.006 \\
\hline FCR & $1.70 \pm 0.05^{\mathrm{ab}}$ & $1.76 \pm 0.03^{a}$ & $1.63 \pm 0.02^{b}$ & 0.009 \\
\hline Fecal score & $2.28 \pm 0.05$ & $2.29 \pm 0.02$ & $2.26 \pm 0.08$ & 0.958 \\
\hline
\end{tabular}

${ }^{1} \mathrm{HWP}$, hydrolyzed wheat protein; FSBM, fermented soybean meal; ESBM, enzyme-treated soybean meal; $B W$, body weight; $A D G$, average daily gain; $A D F I$, average daily feed intake; $F C R$, feed conversion ratio. 
Values are expressed as the means $\pm \operatorname{SEM}, n=6$. ${ }^{\text {a,b }}$ Means within a row with different letters differ at $P<$ 0.05 .

Table 3 The oxidative status in serum of piglets fed diets with different dietary protein sources ${ }^{1}$.

\begin{tabular}{|lllll|}
\hline Item & HWP & FSBM & ESBM & $P$-Value \\
\hline Day 14 & & & \\
\hline AOPP $(\mathrm{pmol} / \mathrm{L})$ & $91.93 \pm 3.37$ & $85.94 \pm 4.60$ & $90.28 \pm 1.93$ & 0.413 \\
\hline FRAP $(\mathrm{mmol} / \mathrm{L})$ & $0.27 \pm 0.01^{\mathrm{b}}$ & $0.26 \pm 0.01^{\mathrm{b}}$ & $0.30 \pm 0.01^{\mathrm{a}}$ & 0.001 \\
\hline MDA $(\mathrm{nmol} / \mathrm{mL})$ & $3.29 \pm 0.10$ & $3.08 \pm 0.23$ & $2.71 \pm 0.13$ & 0.094 \\
\hline SOD $(\mathrm{U} / \mathrm{mL})$ & $127.19 \pm 2.80^{\mathrm{b}}$ & $132.59 \pm 4.25^{\mathrm{b}}$ & $142.89 \pm 2.28^{\mathrm{a}}$ & 0.017 \\
\hline CAT $(\mathrm{U} / \mathrm{mL})$ & $4.32 \pm 0.31$ & $4.47 \pm 0.31$ & $4.61 \pm 0.20$ & 0.746 \\
\hline GSH-Px $(\mathrm{U} / \mathrm{mL})$ & $300.53 \pm 13.29$ & $309.12 \pm 14.49$ & $307.72 \pm 17.08$ & 0.930 \\
\hline Day 28 & & & & \\
\hline AOPP $(\mathrm{pmol} / \mathrm{L})$ & $79.95 \pm 6.66$ & $78.55 \pm 3.60$ & $82.65 \pm 8.90$ & 0.246 \\
\hline FRAP $(\mathrm{mmol} / \mathrm{L})$ & $0.24 \pm 0.01^{\mathrm{b}}$ & $0.24 \pm 0.01^{\mathrm{b}}$ & $0.27 \pm 0.01^{\mathrm{a}}$ & 0.013 \\
\hline MDA $(\mathrm{nmol} / \mathrm{mL})$ & $2.91 \pm 0.15$ & $3.13 \pm 0.09$ & $2.72 \pm 0.15$ & 0.211 \\
\hline SOD $(\mathrm{U} / \mathrm{mL})$ & $138.87 \pm 2.71^{\mathrm{ab}}$ & $133.67 \pm 4.25^{\mathrm{b}}$ & $145.64 \pm 2.28^{\mathrm{a}}$ & 0.039 \\
\hline CAT $(\mathrm{U} / \mathrm{mL})$ & $4.48 \pm 0.37$ & $4.42 \pm 0.30$ & $4.70 \pm 0.36$ & 0.838 \\
\hline GSH-Px $(\mathrm{U} / \mathrm{mL})$ & $303.16 \pm 14.65$ & $262.46 \pm 22.45$ & $290.53 \pm 10.28$ & 0.283 \\
\hline
\end{tabular}

${ }^{1}$ HWP, hydrolyzed wheat protein; FSBM, fermented soybean meal; ESBM, enzyme-treated soybean meal; AOPP, advanced oxidation protein products; FRAP, ferric reducing ability of plasma; MDA, malondialdehyde; SOD, total superoxide dismutase; CAT, catalase; GSH-Px, glutathione peroxidase. Values are expressed as the means $\pm \mathrm{SEM}, n=6$. ${ }^{\text {a,b}}$ Means within a row with different letters differ at $P<$ 0.05 .

Table 4 The serum levels of DAO, endotoxin and D-lactate in piglets fed diets with different dietary protein sources ${ }^{1}$. 


\begin{tabular}{|lllll|}
\hline Item & HWP & FSBM & ESBM & P-Value \\
\hline Day 14 & & & & \\
\hline DAO $((\mathrm{U} / \mathrm{L})$ & $17.91 \pm 0.50$ & $17.56 \pm 0.76$ & $16.57 \pm 0.57$ & 0.257 \\
\hline Endotoxin $(\mathrm{EU} / \mathrm{mL})$ & $15.68 \pm 1.06$ & $16.89 \pm 1.27$ & $16.01 \pm 1.01$ & 0.704 \\
\hline D-lactate $(\mu \mathrm{mol} / \mathrm{L})$ & $163.52 \pm 7.70$ & $164.50 \pm 10.38$ & $174.19 \pm 9.44$ & 0.621 \\
\hline Day 28 & & & & \\
\hline DAO $(\mathrm{U} / \mathrm{L})$ & $14.50 \pm 0.37^{\mathrm{a}}$ & $13.23 \pm 0.59^{\mathrm{ab}}$ & $12.75 \pm 0.42^{\mathrm{b}}$ & 0.044 \\
\hline Endotoxin $(\mathrm{EU} / \mathrm{mL})$ & $12.74 \pm 0.52$ & $12.69 \pm 0.49$ & $12.83 \pm 0.56$ & 0.983 \\
\hline D-lactate $(\mu \mathrm{mol} / \mathrm{L})$ & $218.57 \pm 16.89$ & $223.37 \pm 13.25$ & $211.59 \pm 11.21$ & 0.731 \\
\hline
\end{tabular}

${ }^{1}$ HWP, hydrolyzed wheat protein; FSBM, fermented soybean meal; ESBM, enzyme-treated soybean meal; DAO, diamine oxidase. Values are expressed as the means $\pm \operatorname{SEM}, n=6$. ${ }^{a, b}$ Means within a row with different letters differ at $P<0.05$.

Table 5 The inflammatory cytokines and immunoglobulins in serum of piglets fed diets with different dietary protein sources ${ }^{1}$. 


\begin{tabular}{|c|c|c|c|c|}
\hline Item & HWP & FSBM & ESBM & $P$-Value \\
\hline \multicolumn{5}{|l|}{ Day 14} \\
\hline $\mathrm{IL}-1 \beta(\mathrm{pg} / \mathrm{mL})$ & $17.58 \pm 0.53$ & $17.57 \pm 1.05$ & $14.94 \pm 0.20$ & 0.060 \\
\hline IL-6 (pg/mL) & $107.15 \pm 4.09$ & $102.29 \pm 3.56$ & $92.51 \pm 3.52$ & 0.070 \\
\hline TNF-a (pg/mL) & $77.10 \pm 6.63$ & $69.57 \pm 6.76$ & $71.37 \pm 3.49$ & 0.699 \\
\hline $\lg A(g / L)$ & $1.05 \pm 0.08$ & $1.11 \pm 0.05$ & $1.19 \pm 0.08$ & 0.461 \\
\hline $\lg G(g / L)$ & $7.20 \pm 0.61$ & $7.11 \pm 0.44$ & $7.70 \pm 0.76$ & 0.817 \\
\hline $\lg M(g / L)$ & $0.58 \pm 0.03$ & $0.69 \pm 0.07$ & $0.70 \pm 0.05$ & 0.286 \\
\hline \multicolumn{5}{|l|}{ Day 28} \\
\hline $\mathrm{IL}-1 \beta(\mathrm{pg} / \mathrm{mL})$ & $20.20 \pm 0.54^{\mathrm{a}}$ & $18.86 \pm 0.91^{\mathrm{ab}}$ & $16.45 \pm 0.87^{b}$ & 0.027 \\
\hline IL-6 (pg/mL) & $86.33 \pm 7.34$ & $95.23 \pm 6.06$ & $84.96 \pm 6.48$ & 0.624 \\
\hline TNF-a (pg/mL) & $77.91 \pm 5.42$ & $74.91 \pm 3.62$ & $71.52 \pm 5.27$ & 0.694 \\
\hline $\lg A(g / L)$ & $1.21 \pm 0.12$ & $1.22 \pm 0.08$ & $1.16 \pm 0.10$ & 0.775 \\
\hline $\lg G(g / L)$ & $9.52 \pm 0.59$ & $9.92 \pm 0.29$ & $10.30 \pm 0.58$ & 0.474 \\
\hline $\lg M(g / L)$ & $0.71 \pm 0.07$ & $0.77 \pm 0.05$ & $0.83 \pm 0.05$ & 0.409 \\
\hline
\end{tabular}

${ }^{1} \mathrm{HWP}$, hydrolyzed wheat protein; FSBM, fermented soybean meal; ESBM, enzyme-treated soybean meal; IL-1 $\beta$, interleukin-1 $\beta$; IL-6, interleukin-6; TNF-a, tumour necrosis factor-a; IgA, immunoglobulin A; IgG, immunoglobulin $\mathrm{G}$; IgM, immunoglobulin $\mathrm{M}$. Values are expressed as the means $\pm \mathrm{SEM}, n=6$. ${ }^{\mathrm{a}, \mathrm{b}}$ Means within a row with different letters differ at $P<0.05$.

Table 6 The concentrations of SCFAs and BCFAs in feces of piglets fed diets with different dietary protein sources ${ }^{1}$. 


\begin{tabular}{|lllll|}
\hline Item, mg/g & HWP & FSBM & ESBM & $P$-Value \\
\hline Acetate & $5.68 \pm 0.42$ & $5.27 \pm 0.38$ & $5.74 \pm 0.36$ & 0.625 \\
\hline Propionate & $3.19 \pm 0.29$ & $2.68 \pm 0.24$ & $2.95 \pm 0.15$ & 0.359 \\
\hline Butyrate & $1.71 \pm 0.10^{\mathrm{ab}}$ & $1.59 \pm 0.07^{\mathrm{b}}$ & $1.94 \pm 0.10^{\mathrm{a}}$ & 0.029 \\
\hline SCFAs & $10.59 \pm 0.74$ & $9.54 \pm 0.59$ & $10.63 \pm 0.52$ & 0.375 \\
\hline Isobutyrate & $0.37 \pm 0.03$ & $0.30 \pm 0.04$ & $0.37 \pm 0.03$ & 0.247 \\
\hline Isovalerate & $0.39 \pm 0.04$ & $0.32 \pm 0.05$ & $0.37 \pm 0.03$ & 0.559 \\
\hline Valerate & $0.58 \pm 0.05$ & $0.51 \pm 0.09$ & $0.49 \pm 0.08$ & 0.702 \\
\hline BCFAs & $1.35 \pm 0.06$ & $1.13 \pm 0.17$ & $1.23 \pm 0.14$ & 0.577 \\
\hline Total SCFAs & $11.93 \pm 0.78$ & $10.67 \pm 0.71$ & $11.85 \pm 0.64$ & 0.342 \\
\hline
\end{tabular}

${ }^{1} \mathrm{HWP}$, hydrolyzed wheat protein; FSBM, fermented soybean meal; ESBM, enzyme-treated soybean meal. Values are expressed as the means $\pm \mathrm{SEM}, n=6$. ${ }^{\text {a,b }}$ Means within a row with different letters differ at $P<$ 0.05 .

Table 7 The concentrations of $\mathrm{NH}_{3}-\mathrm{N}$ and biogenic amine in feces of piglets fed diets with different dietary protein sources ${ }^{1}$.

\begin{tabular}{|lllll|}
\hline Item, $\mu \mathrm{g} / \mathrm{g}$ & HWP & FSBM & ESBM & $P$-Value \\
\hline $\mathrm{NH}_{3} \mathrm{~N}$ & $151.56 \pm 15.10$ & $154.35 \pm 10.93$ & $162.49 \pm 11.14$ & 0.827 \\
\hline Putrescine & $3.33 \pm 0.28$ & $3.19 \pm 0.50$ & $2.10 \pm 0.48$ & 0.267 \\
\hline Cadaverine & $3.08 \pm 0.43$ & $3.21 \pm 0.46$ & $2.01 \pm 0.26$ & 0.076 \\
\hline Spermine & $0.25 \pm 0.02$ & $0.19 \pm 0.05$ & $0.27 \pm 0.03$ & 0.406 \\
\hline
\end{tabular}

${ }^{1} \mathrm{HWP}$, hydrolyzed wheat protein; FSBM, fermented soybean meal; ESBM, enzyme-treated soybean meal. Values are expressed as the means $\pm \mathrm{SEM}, n=6$.

\section{Figures}



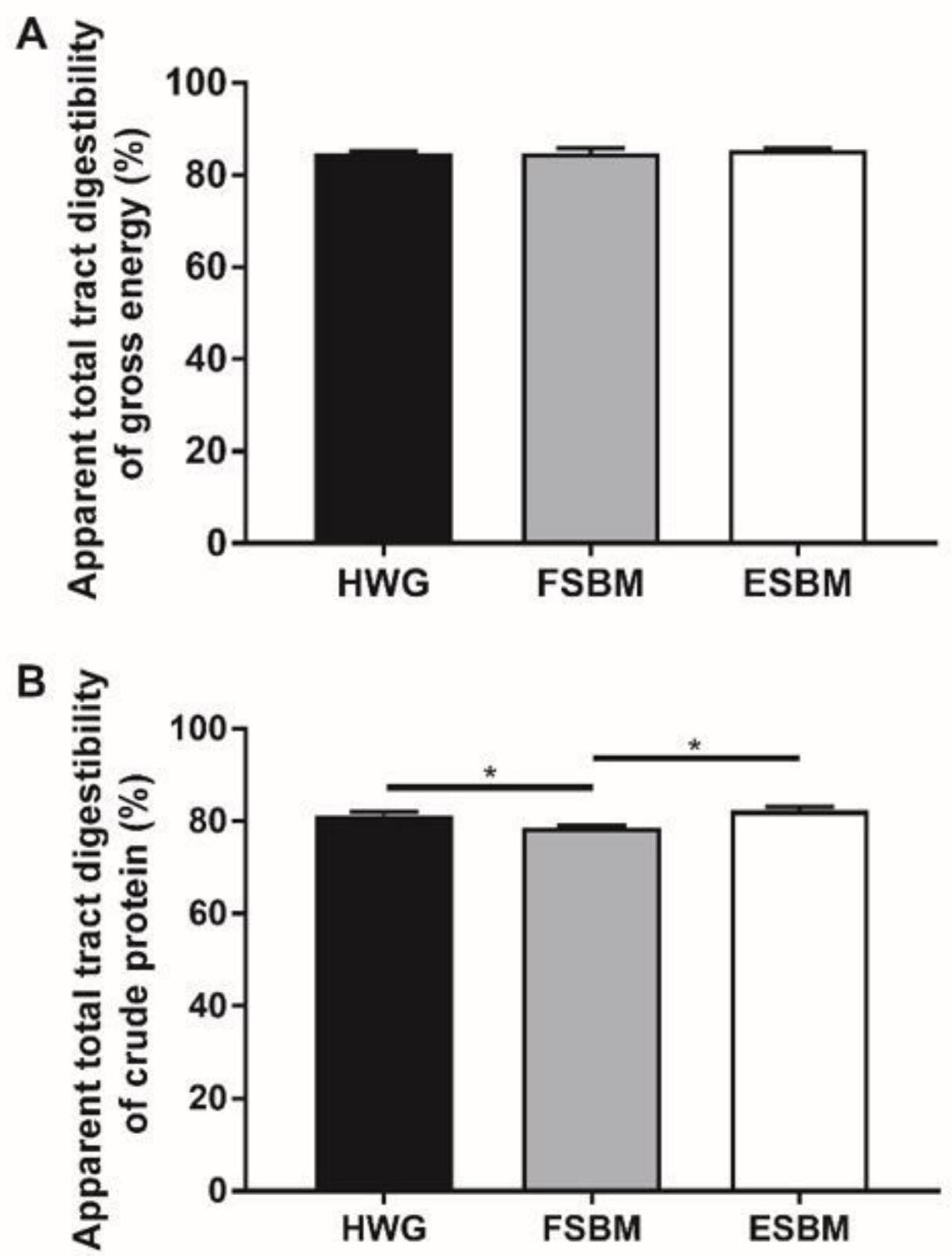

Figure 1

The apparent total tract digestibility of nutrients in piglets fed diets with different dietary protein sources. (A) Gross energy. (B) Crude protein. HWP, hydrolyzed wheat protein; FSBM, fermented soybean meal; ESBM, enzyme-treated soybean meal. * $\mathrm{P}<0.05$ means significant difference. 

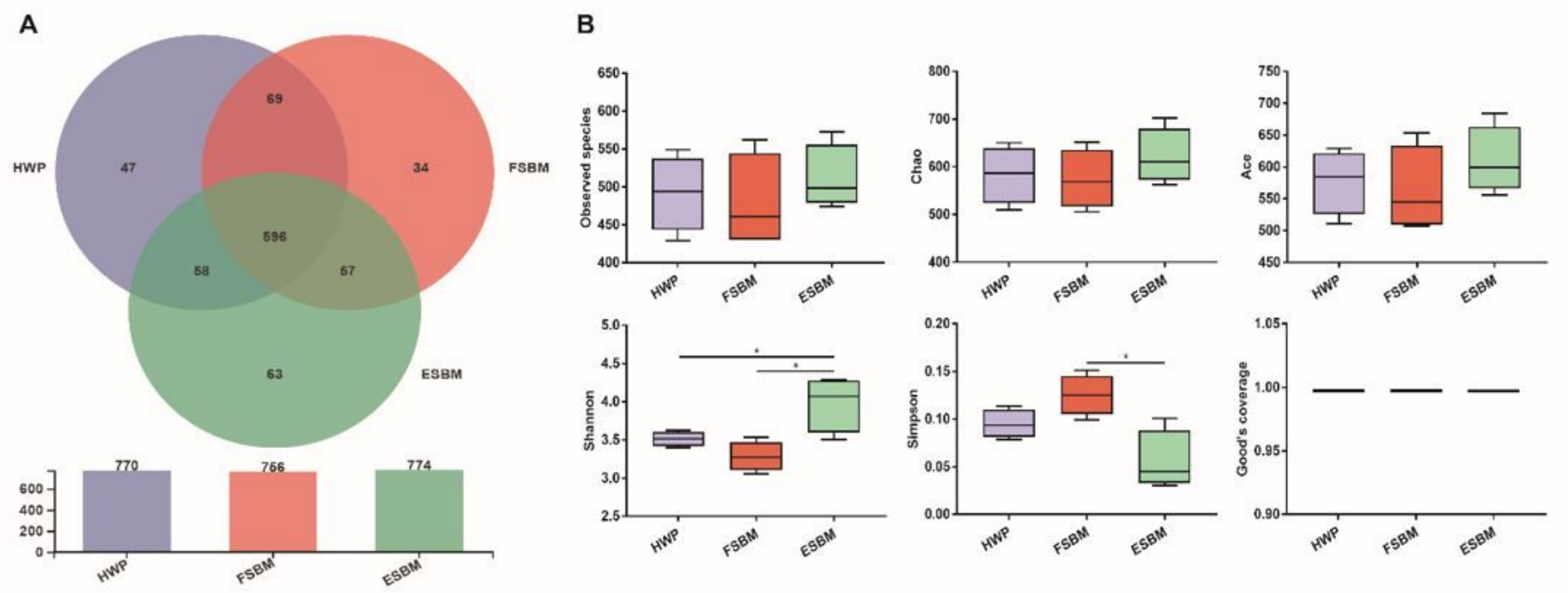

\section{Figure 2}

Fecal microbiota richness and diversity in piglets fed diets with different dietary protein sources. (A) OUT Venn of three dietary treatments. (B) Comparison of a-diversity indices among three dietary treatments. HWP, hydrolyzed wheat protein; FSBM, fermented soybean meal; ESBM, enzyme-treated soybean meal. *P $<0.05$ means significant difference.

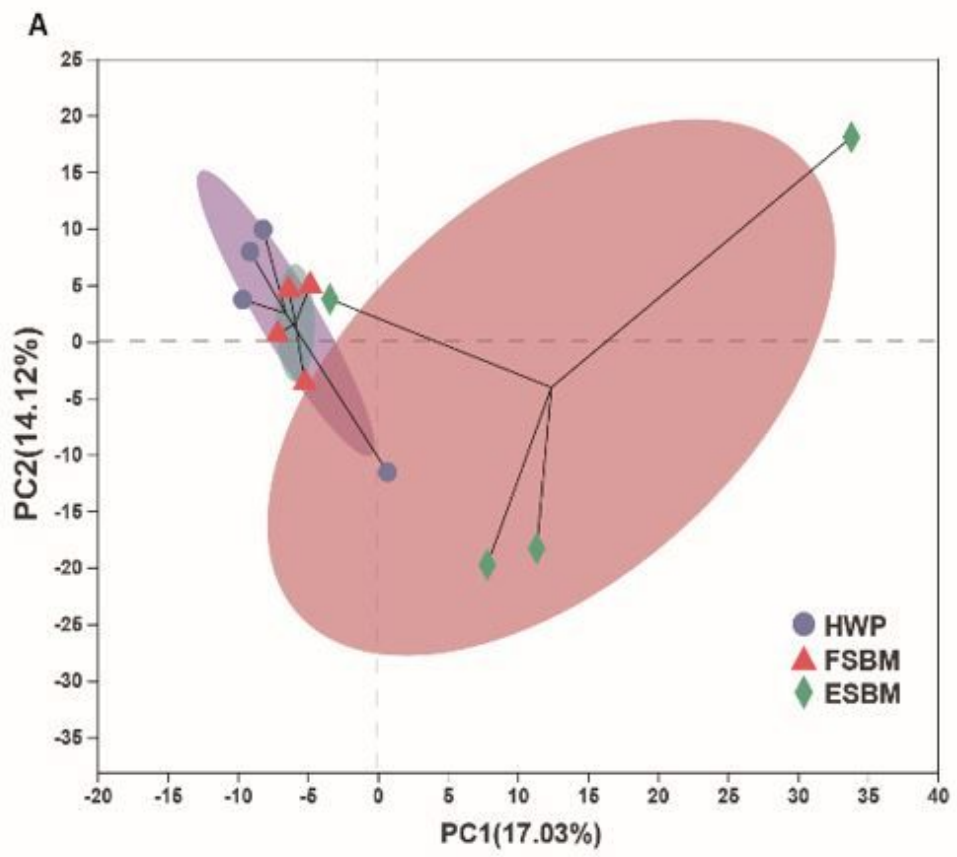

B

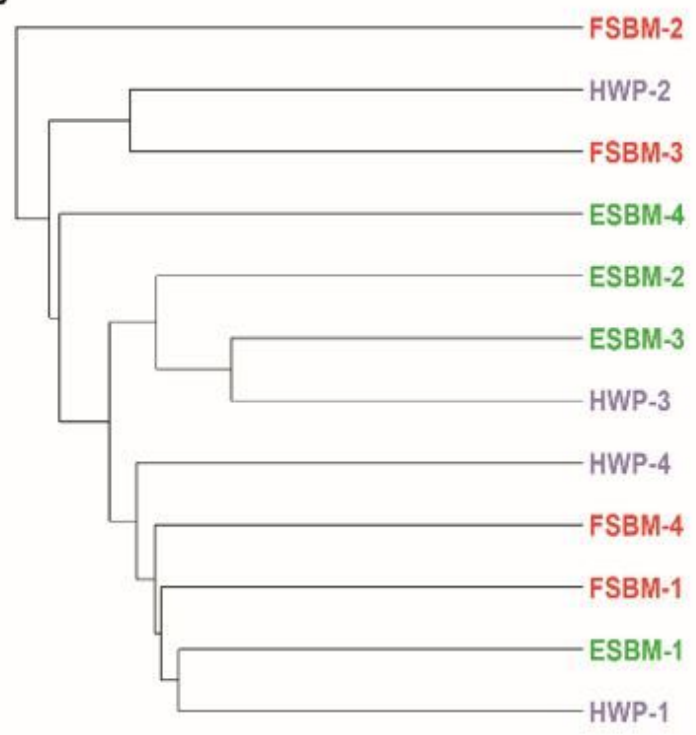

\section{Figure 3}

Comparison of fecal microbiota structure by $\beta$-diversity in piglets fed diets with different dietary protein sources. (A) Principal component analysis (PCA) of the microbiota communities based on the OUT level. 
(B) UPGMA tree, all revealing significant differences among the different treatments based on Unweighted UniFrac distances of the OTU community. HWP, hydrolyzed wheat protein; FSBM, fermented soybean meal; ESBM, enzyme-treated soybean meal.
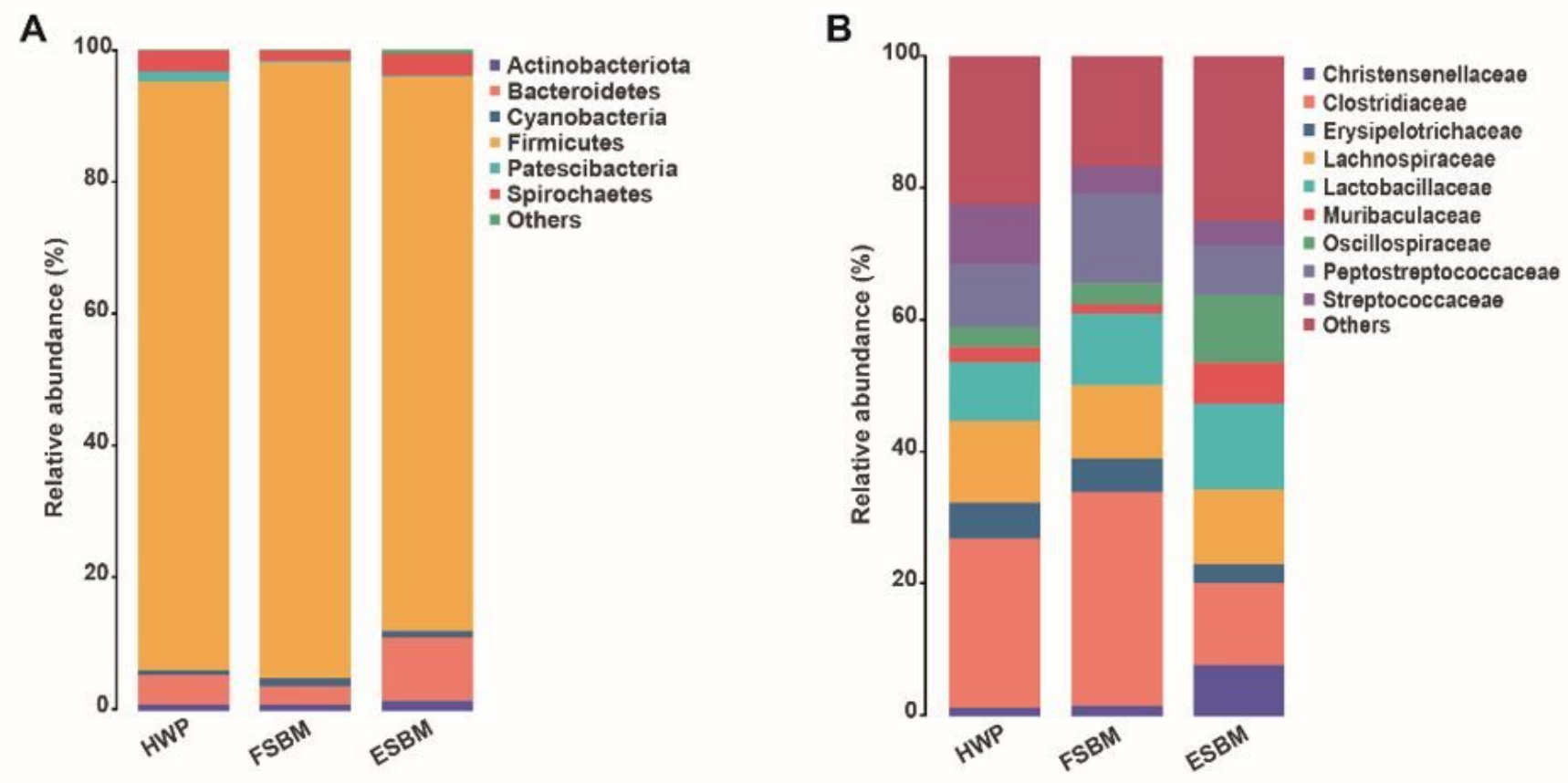

\section{Figure 4}

Relative abundance of fecal microbiota in piglets fed diets with different dietary protein sources. (A) Relative abundance of microbial community at the phylum level with the abundance higher than $0.01 \%$. (B) Relative abundance of microbial community at the family level with the abundance higher than $0.05 \%$. HWP, hydrolyzed wheat protein; FSBM, fermented soybean meal; ESBM, enzyme-treated soybean meal. 
A

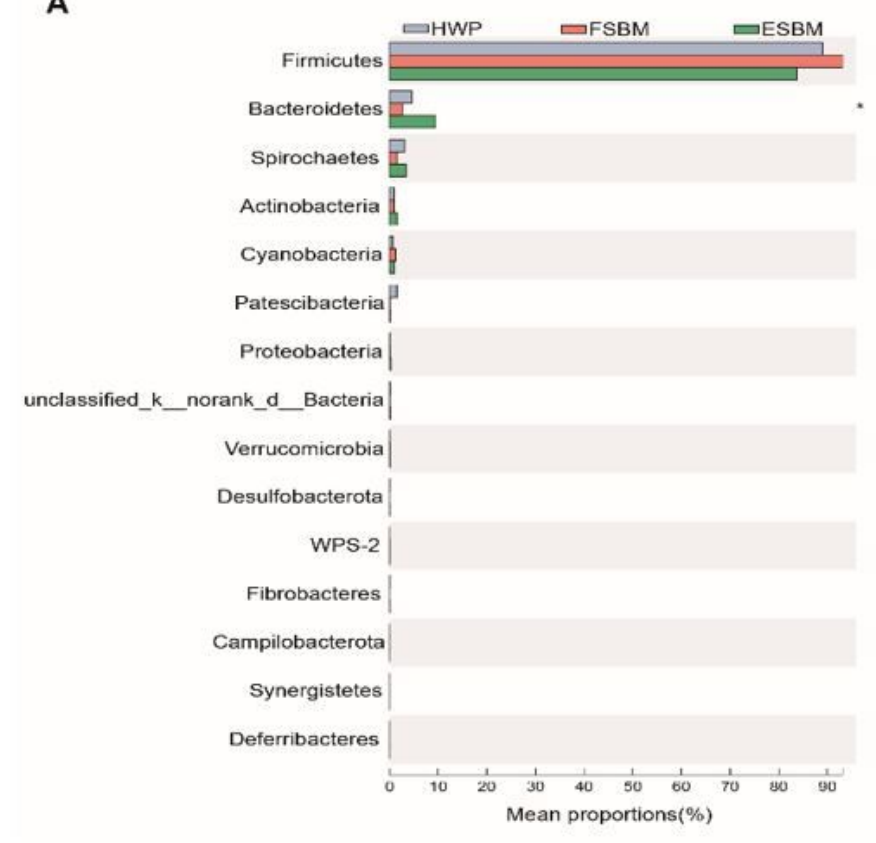

B

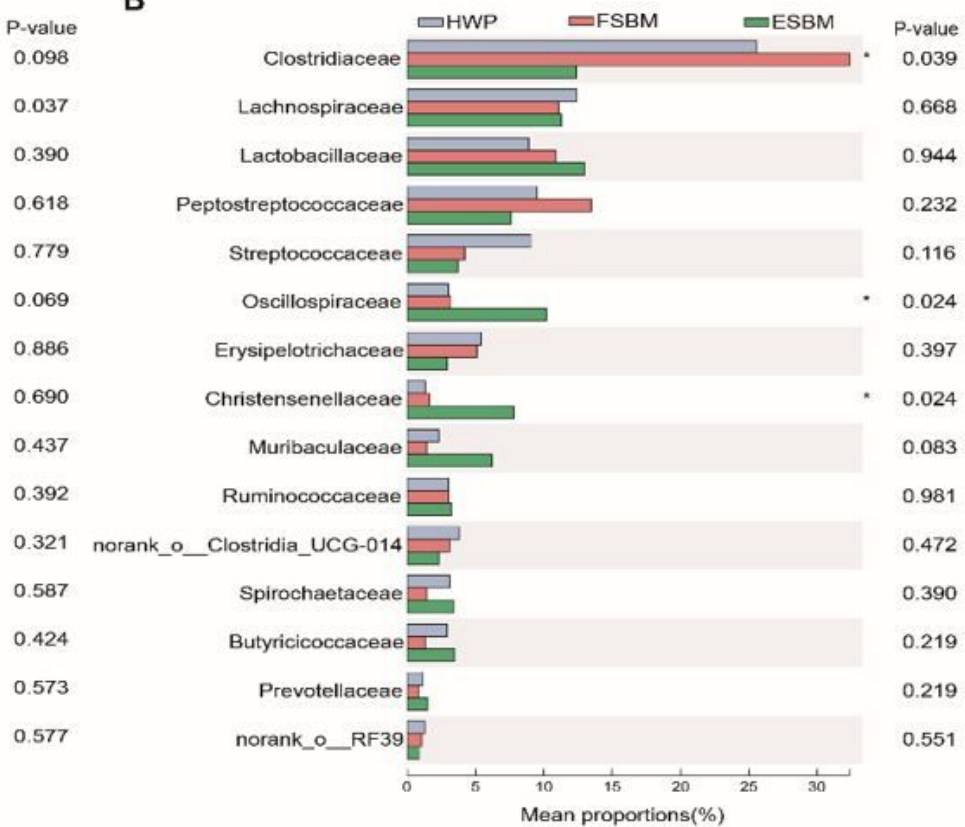

\section{Figure 5}

Differences in the fecal microbiota compositions of piglets fed diets with different dietary protein sources. (A) Structural differentiation of the fecal microbiota at the phylum level based on a contribution degree at top 15. (B) Structural differentiation of the fecal microbiota at the family level based on a contribution degree at top 15. HWP, hydrolyzed wheat protein; FSBM, fermented soybean meal; ESBM, enzyme-treated soybean meal. ${ }^{\star} \mathrm{P}<0.05$ means significant difference.

A

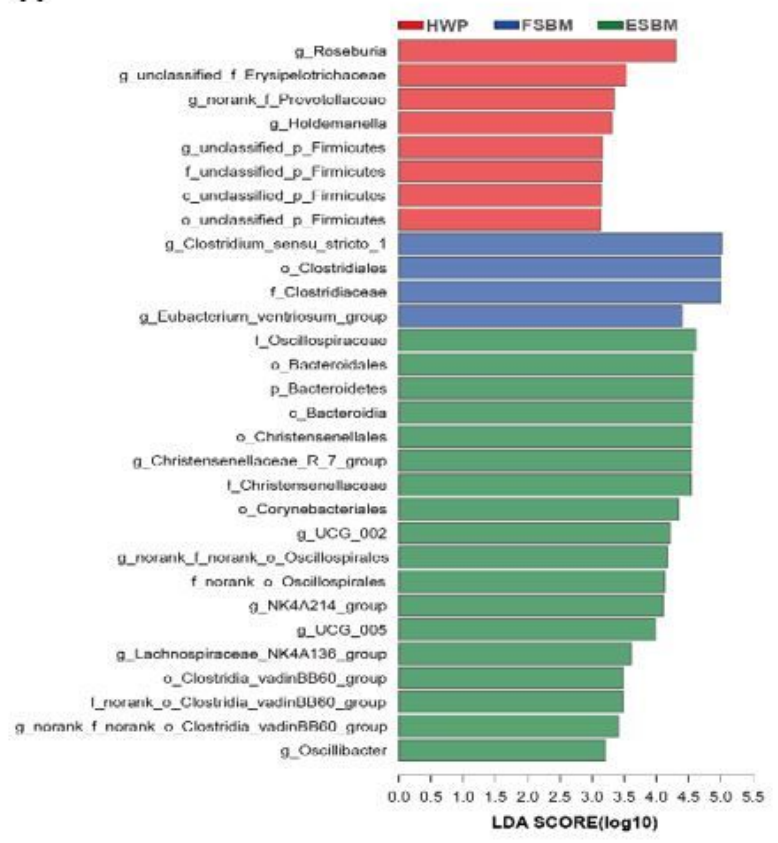

B

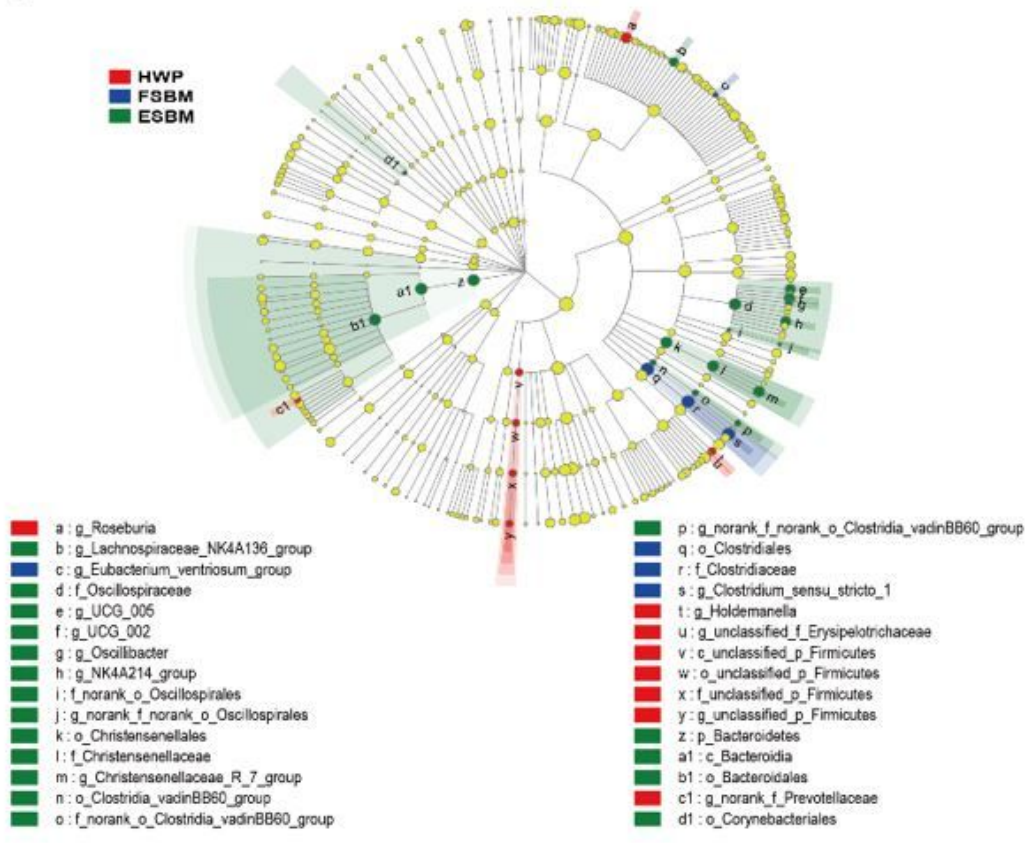




\section{Figure 6}

LefSE analysis of fecal microbiota among three dietary treatments. (A) Linear discriminant analysis (LDA) score of the fecal microbiota among three dietary treatments. An LDA score of $\geq 3$ was considered significant. (B) Cladogram of LEfSe represents taxonomic profiling for the distinct bacteria from the phylum to genus level, the node size means the average relative abundance of the taxon, the yellow node means no difference among different treatment, but the other color nodes means the significant difference. HWP, hydrolyzed wheat protein; FSBM, fermented soybean meal; ESBM, enzyme-treated soybean meal.
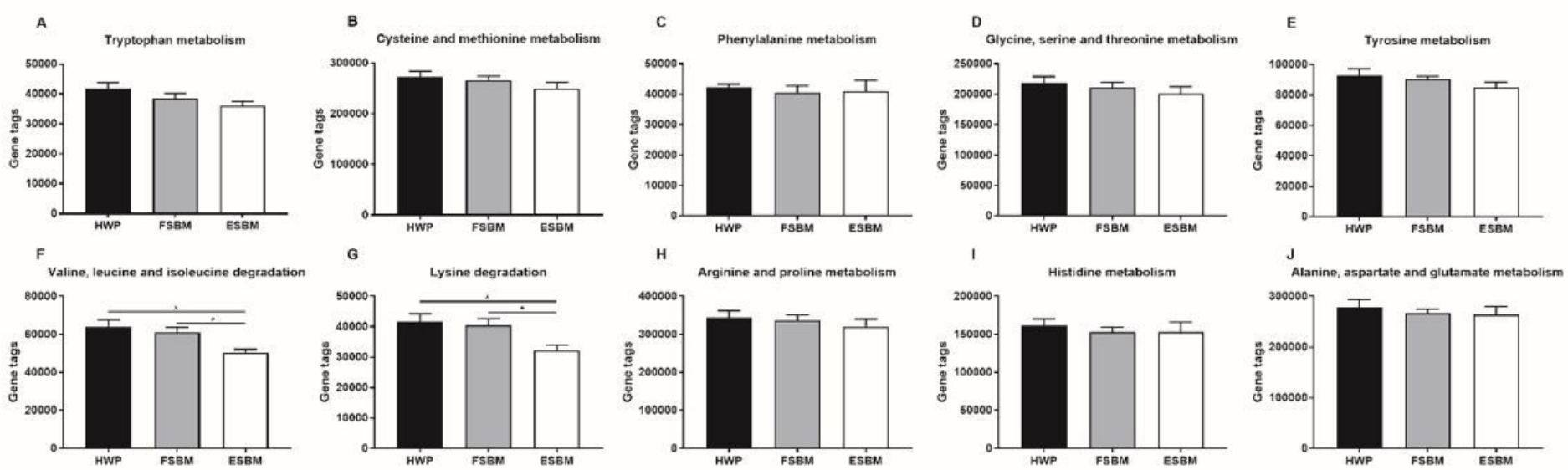

Figure 7

Prediction on amino acid metabolism of bacterial communities using the PICRUSt program. (A) Tryptophan metabolism. (B) Cysteine and methionine metabolism. (C) Phenylalanine metabolism. (D) Glycine, serine and threonine metabolism. (E) Tyrosine metabolism. (F) Valine, leucine and isoleucine degradation. (G) Lysine degradation. (H) Arginine and proline metabolism. (I) Histidine metabolism. (J) Alanine, aspartate and glutamate metabolism. HWP, hydrolyzed wheat protein; FSBM, fermented soybean meal; ESBM, enzyme-treated soybean meal. * $\mathrm{P}<0.05$ means significant difference. 